\title{
Outcomes of infants born to mothers receiving methadone for pain management in pregnancy
}

\author{
C Sharpe, C Kuschel
}

See end of article for authors' affiliations

.....................

Correspondence to: Dr Kuschel, Newborn Services, National

Women's Hospital, Private

Bag 92 189, Auckland New Zealand;

CarlK@adhb.govt.nz

Accepted

13 February 2003 Objective: To assess the outcomes of infants born to mothers receiving methadone for the management of
pain in pregnancy. Design: A retrospective audit was conducted of neonatal outcomes in 19 cases in which methadone was used in the management of maternal pain (pain group). Twenty four cases in which methadone was used for maintenance of opiate addiction in pregnancy served as a comparison group (maintenance group). Setting: Regional level 3 neonatal service.

Results: Infants in the pain group were exposed to significantly smaller methadone doses, for shorter periods, starting later in pregnancy. Only $11 \%$ of them required treatment for neonatal abstinence syndrome, whereas $58 \%$ of infants in the maintenance group required treatment. Other neonatal morbidity in the pain group was considerable, probably related to prematurity. Infants in the pain group had significantly higher $z$ scores for birth weight and head circumference, but not length, than the infants in the maintenance group.

Conclusions: Methadone used for the treatment of maternal pain resulted in a low incidence of neonatal abstinence syndrome. Infants were normally grown. However, there was significant morbidity related to slight prematurity, and delivery in this group should be delayed until term if possible.
$T$ he treatment of acute or chronic pain syndromes in pregnant women poses some challenging problems. When pain is poorly controlled, the mother may be placed at risk from prolonged bed rest and immobility, self medication, and the adverse psychological effects of uncontrolled pain. However, pharmacological treatment can pose risks to the fetus both through direct effects on a developing organism and the possibility of fetal habituation to drugs.

At the National Women's Hospital, a strategy using methadone (a long acting opioid) has been used for women presenting with serious pain problems during pregnancy not responsive to non-pharmacological treatment or local pharmacotherapy. Despite extensive experience of the use of methadone for management of opiate addiction during pregnancy, there are few data on its use in pregnancy for treatment of chronic pain. Methadone for management of addiction often results in neonatal abstinence syndrome (NAS). Experience with methadone for management of addiction has also raised concerns about the adverse effect of methadone on infant birth weight, head circumference, and subsequent development. ${ }^{1-8}$

We performed a retrospective audit of infants exposed in utero to methadone administered for the treatment of maternal pain and compared the outcomes for these infants with a group of infants born to mothers who were managed with methadone in pregnancy for opiate addiction.

\section{METHODS}

Cases were identified retrospectively from a database kept by the Pain Service at the National Women's Hospital. Between August 1997 (when this strategy was first introduced) and November 2000, 19 mothers were identified in whom methadone was used for pain management (designated the pain group). Hospital records were reviewed to examine outcomes in all cases. A further 24 women in whom methadone was used for maintenance of opiate addiction from August 1999 to May 2001 were used as a comparison group (designated the maintenance group). Data were prospectively collected on this group as part of another study.

The pain management strategy has been described elsewhere. ' Initially non-pharmacological treatments, such as heat, physiotherapy, transcutaneous electrical nerve stimulation, acupuncture, hypnotherapy, and psychosocial support, and local pharmacotherapy through anaesthetic and steroid injections of trigger points or clonidine patches were prescribed for pain problems. When this treatment was unable to control pain, systemic pharmacotherapy was added in three steps. Patients were first treated with regular paracetamol, then non-opiate systemic pharmacotherapy tailored to the type of pain involved. Some patients received diclofenac for severe musculoskeletal pain. Amitriptyline was used to treat neuropathic pain and visceral spasm and as headache prophylaxis. Mexiletine was also used to treat neuropathic pain. Orphenadrine was used in rare instances to treat skeletal muscle spasm. When maternal pain remained uncontrolled, patients received morphine or pethidine. When a long term requirement for opiates was identified, methadone was started in addition to the drugs noted above, and the short acting opiates were discontinued.

Maternal data collected were: age, indication for treatment, comorbidities, smoking status, other medication use, gestation at start of methadone treatment, highest methadone dose, dose at delivery, and length of methadone treatment. Adverse events of pregnancy and delivery recorded in the antenatal chart were collected. These included the occurrence of intrauterine growth retardation, antepartum haemorrhage, gestational proteinuric hypertension, and preterm delivery (less than 37 weeks gestation).

Neonatal data collected included gestation, growth parameters, condition at birth, withdrawal symptoms (including scores from modified Finnegan scores ${ }^{10}$ ), requirement for treatment of withdrawal and duration of treatment, length of stay, and any other adverse neonatal events such as jaundice, feeding problems, and sepsis. 
Birth weight, head circumference, and length measurements were converted into standard deviation ( $\mathrm{z}$ score) values for gestation and sex, using population based data for New South Wales. ${ }^{11}$ z Scores are calculated as (individual value minus population mean) divided by population standard deviation.

Continuous variables and nominal variables were analysed using the Wilcoxon rank-sum test and $\chi^{2}$ test respectively with the statistical software package SAS (release 8.2; SAS Institute Inc, Cary, North Carolina, USA).

\section{RESULTS}

\section{Maternal characteristics}

Women in the pain group had a diverse range of diagnoses. In many cases chronic pain preceded pregnancy, but was exacerbated by the pregnancy. Indications for treatment included chronic pelvic pain (caused by pelvic arthropathy or a road traffic accident) in seven women, abdominal pain (caused by renal colic, nerve entrapment, biliary colic, or degenerating fibroid) in six patients, and back pain (disc prolapse and, in one case, related to a phaeochromocytoma) in six patients.

Comorbidities in the pain group included hypertension (two), asthma (six), and treated cervical intraepithelial neoplasia (two). Two women had depression, and two had a history of alcohol or drug abuse. The smoking rate in the maintenance group was high $(\mathrm{n}=21)$, as found almost universally in other studies of women managed with methadone for addiction. ${ }^{12}$ Nine women in the pain group smoked during pregnancy.

Continued use of illicit drugs was admitted by some patients in the maintenance group and may have been present in others. Four women were known to continue to use intravenous opioids, eight were known to use cannabis, and five were receiving prescribed benzodiazepines. None admitted to use of alcohol in pregnancy. There was no documented use of illicit drugs in the pain group, although one woman used prescribed benzodiazepines and one admitted to occasional use of alcohol in pregnancy.

The use of many drugs in the pain group within and outside the pain treatment strategy was striking. All women in the pain group were prescribed regular paracetamol and amitriptyline, 14 received clonidine at some stage, 13 received mexiletine, five received diclofenac, and one received orphenadrine. In many cases, oral and intravenously administered morphine preparations were used for brief periods before methadone treatment was started. Other medicines outside the pain treatment strategy included cyclizine, methyldopa, codeine, dexamethasone, albuterol, becotide, nortriptyline, fluoxetine, clomiphene, promethazine, doxazosin, chloral hydrate, and antibiotics.

The use of methadone differed significantly between the two groups. Women in the pain group started methadone later (median gestation 30 weeks (range 0-35) $v 0$ weeks (range 0-33), p $<0.0001$ ), at a lower maximum dose (median $40 \mathrm{mg}$ (range 10-180) v $60 \mathrm{mg}$ (range 23-105), $\mathrm{p}=0.0037$ ), and for a shorter duration ( 5 weeks (range $1-$ 36) $v 36$ weeks (range 6-41), p $<0.0001$ ) than the maintenance group.

In the pain group, two cases of intrauterine growth retardation were identified during pregnancy by obstetricians. In the maintenance group, four cases were identified. Two women in the pain group required antihypertensive treatment for gestational proteinuric hypertension, one woman in the context of pre-existing chronic hypertension. Three pregnancies in the pain cohort were complicated by antepartum haemorrhage. However, in the one case of abruption and in one other case of antepartum haemorrhage, haemorrhage predated, and hence was not attributable to, the systemic pharmacotherapy.

\section{Neonatal outcomes}

There was a high rate of slight prematurity in the pain group, in whom 11 infants were delivered before 37 weeks. The median gestation at delivery was 36 weeks (range 32-41), with a high rate of preterm delivery between 36 and 37 weeks. Delivery of nine infants was induced, with uncontrolled maternal pain commonly being cited as one indication for delivery, although in three cases it was the sole indication. In four cases, there was spontaneous onset of preterm labour or spontaneous preterm rupture of membranes leading to induction. Ten infants in the pain group were born by spontaneous vaginal delivery, three by elective caesarean section, three by emergency caesarean section, and three required assisted delivery.

In contrast, infants in the maintenance group had a significantly greater median gestation at delivery of 39 weeks (range $35-41)(p=0.0002)$. In the three cases of preterm delivery, infants were induced because of intrauterine growth retardation. In this group, 17 infants were born by spontaneous vaginal delivery, one was delivered by breech delivery, there were four emergency caesareans, and three assisted deliveries.

Infants in both groups were generally in good condition at birth and required little or no resuscitation. The median one minute Apgar scores were 9 in both groups, and five minute Apgar scores were not significantly different. Infants in the pain group had significantly higher median $\mathrm{z}$ scores for birth weight $(-0.04 v-0.69, \mathrm{p}=0.025)$ and head circumference $(+0.75 v-0.75, \mathrm{p}=0.0009)$ than the maintenance group. z Scores for length did not differ significantly between the two groups (fig 1).

Thirteen of the infants in the pain group and all the infants in the maintenance group exhibited some withdrawal symptoms when scored against standardised protocols. ${ }^{10}$ Routine toxicology screens of urine or meconium were not performed on either group unless there were concerns about undisclosed maternal illicit drug use. Fourteen infants (58\%) in the maintenance group had withdrawal symptoms severe

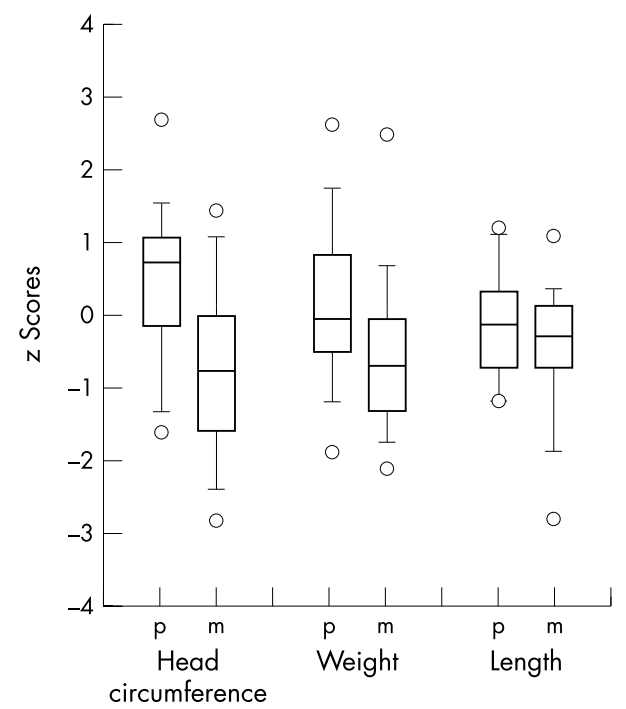

Figure 1 Growth parameters expressed as $\mathrm{z}$ scores for infants from pregnancies in which methadone was used for pain ( $p$ ) or for maintenance of opiate addiction $(\mathrm{m})$. The boxes show $25 \mathrm{th}-75$ th centiles, whiskers show 10th-90th centiles, and circles show 5th-95th centiles. 
enough to require treatment. In contrast, only two infants $(11 \%)$ in the pain group required treatment $(p=0.0016)$. One of these infants had severe persistent pulmonary hypertension of the newborn and was treated with morphine for sedation while ventilated, but required prolonged treatment because of withdrawal symptoms when this was initially ceased.

Three infants in the pain group received assisted ventilation with continuous positive airway pressure for respiratory distress, five developed jaundice requiring phototherapy, and nine had feeding problems, which in two cases was a prolonged problem. One infant required admission to the newborn unit for hypoglycaemia. One infant who developed group B streptococcal sepsis was induced at 37 weeks because of maternal pain secondary to biliary colic; there were no identified maternal risk factors for sepsis. One infant who had been prenatally diagnosed with a cervical meningocele before the initiation of maternal pain treatment required intensive care.

One infant developed severe persistent pulmonary hypertension of the newborn. This infant had been exposed to mexilitene, amitriptyline, and methadone for one week before delivery. His mother was induced because of poorly controlled pain from pelvic arthropathy. She went on to have a normal vaginal delivery of a male infant with Apgar scores of 6 and 7; he required positive pressure ventilation for one minute. The infant was initially well enough to go to the postnatal ward, but later deteriorated requiring full intensive care and, as noted above, elicited signs of withdrawal on weaning of a morphine infusion.

In contrast, the infants born to mothers receiving methadone for addiction had very little morbidity. The most noteworthy event was one infant who required a partial exchange transfusion for symptomatic polycythaemia.

The rates of successful breast feeding were high in both groups. In the pain group, 15 infants were breast fed on discharge, and four were receiving bottle feeds only. In the maintenance group, 18 infants were breast fed, and four were receiving bottle feeds only. In two cases, feeding method was unknown.

\section{DISCUSSION}

This audit shows that infants born to mothers receiving methadone for the treatment of chronic pain conditions in pregnancy have little morbidity directly associated with methadone use.

The rates of withdrawal requiring treatment were lower in the infants in the pain group than in the comparative group of infants delivered to women receiving methadone for the treatment of addiction. This is important as there is a need for opiate analgesia in severe pain problems in pregnancy, and anecdotal experience with short acting opiates in our institution has been that withdrawal was often severe and prolonged. The lower doses and shorter treatment periods in the pain group may account for this decreased incidence of NAS, although this is controversial. Some studies have found an association between higher maternal methadone dose and severity of NAS, whereas others argue that there is no such relation. ${ }^{72-14}$ In this study, the percentage of infants born to mothers receiving methadone for treatment of addiction who required treatment for withdrawal is in line with experience elsewhere with methadone used for management of addiction. ${ }^{8}$ The severity of NAS may also have been modified by the increased incidence of preterm delivery in the pain group. Preterm delivery is associated with less severe NAS requiring treatment, although this reduction in incidence is less apparent in the mildly preterm population (such as in this study). ${ }^{15}$
Despite the small numbers in the group, it is somewhat reassuring that the pain group had growth parameters significantly better than infants in the maintenance group, and not significantly smaller than the New South Wales population data. Although the New South Wales data are derived from a different population, they are the most appropriate data available. Better growth may be accounted for by the lower doses and shorter duration of treatment used in the pain group. However, as with NAS, there are conflicting results from previous studies investigating whether a dose-response relation exists between methadone in pregnancy and growth retardation. ${ }^{37813}$ It may also reflect the fact that the pain group generally received treatment after the first trimester, a period when drug exposure would be more likely to produce symmetrical growth retardation and affect brain development. ${ }^{2} 37$

In the literature on methadone maintenance, it is acknowledged that poor growth may in part be due to confounding problems of poor maternal health and nutrition, other drug use, smoking, and poor socioeconomic status. ${ }^{2}{ }^{4} 12$ These problems were less common in the pain group, which may also account for their more normal fetal growth. It is extremely difficult to separate these confounding problems from the methadone effect in the maintenance population, thus our data-from women on methadone without these confounders-may be of interest.

The infant morbidity seen in the pain group probably relates to their slight prematurity. This may indicate a need to delay elective preterm delivery even in mothers with poorly controlled pain. The case of persistent pulmonary hypertension of the newborn requiring intensive care support was unexpected, and an association between in utero drug exposure and the development of this condition cannot be excluded.

This audit does not look at long term outcome for infants exposed to methadone in utero. Matters of concern in the long term of exposed infants include both their neurodevelopmental outcome and predisposition to drug addiction in adulthood. ${ }^{126-816}$ As with almost all drugs, safety information on drugs used in the management of pain in pregnancy is lacking. We continue to monitor closely the use of methadone and the current pain treatment strategy of our institution.

\section{ACKNOWLEDGEMENTS}

We thank the National Women's Hospital Pain Service for their assistance with identifying the infants in this audit.

\section{Authors' affiliations}

C Sharpe, C Kuschel, Newborn Services, National Women's Hospital, Private Bag 92 189, Auckland, New Zealand

\section{REFERENCES}

1 Coles CD, Platzman KA. Behavioral development in children prenatally exposed to drugs and alcohol. Int J Addict 1993;28:1393-433.

2 Behnke M, Eyler FD. The consequences of prenatal substance use for the developing fetus, newborn, and young child. Int J Addict 1993;28:1341-91.

3 Doberczak TM, Thornton JC, Bernstein J, et al. Impact of maternal drug dependency on birth weight and head circumference of offspring. American Journal of Diseases in Children 1987;141:1163-7.

4 Lifschitz MH, Wilson GS, O'Brian Smith E, et al. Factors affecting head growth and intellectual function in children of drug addicts. Pediatrics 1985:75:269-74.

5 Soepatmi S. Developmental outcomes of children of mothers dependent on heroin or heroin/methadone during pregnancy. Acta Paediatr 1994;(suppl 404):36-9.

6 Van Baar AL, Soepatmi S, Gunning WB, et al. Development after prenatal exposure to cocaine, heroin and methadone. Acta Paediatr 1994; (suppl 404):40-6.

7 Wouldes TA. Methadone maintenance during pregnancy: the consequences of low dose vs. high dose for the foetus, the neonate and the infant. PhD thesis. University of Auckland, 2001. 
8 Zuckerman B, Bresnahan K. Development and behavioural consequences of prenatal drug and alcohol exposure. Pediatr Clin North Am $1991 ; 38: 1387-406$

9 Roche S, Hughes EW. Pain problems associated with pregnancy and their management. Pain Reviews 1999:6:239-61.

10 Finnegan LP. Neonatal abstinence syndrome: assessment and pharmacotherapy. In: Rubaltelli FF, e, eds. Neonatal therapy: an update. New York: Elsevier Science Publishers, 1986:122-46.

11 Beeby PJ, Bhuta T, Taylor LK. New South Wales population-based birthweight percentile charts. J Paediatr Child Health 1996;32:512-18.

12 Ward J, Mattick RP, Hall W. Methadone maintenance during pregnancy. In: Ward J, Mattick RP, Hall W, eds. Methadone maintenance treatment and other opioid replacement therapies. Amsterdam: Harwood Academic, 1998:397-417.

13 Suffet F, Brotman R. A comprehensive care program for pregnant addicts: obstetrical, neonatal and child development outcomes. Int J Addict 1984;19:199-219.

14 Mack G. Thomas D, Giles W et al. Methadone levels and neonatal withdrawal. J. Paediatr Child Health 1991;27:96-100

15 Doberczak TM, Kandall SR, Wilets I. Neonatal opiate abstinence syndrome in term and preterm infants. J Pediatr 1991:118:933-7.

16 Jacobson B, Nyberg K, Gronbladh L, et al. Opiate addiction in adult offspring through possible imprinting after obstetric treatment. BMJ 1990;301:1067-70.

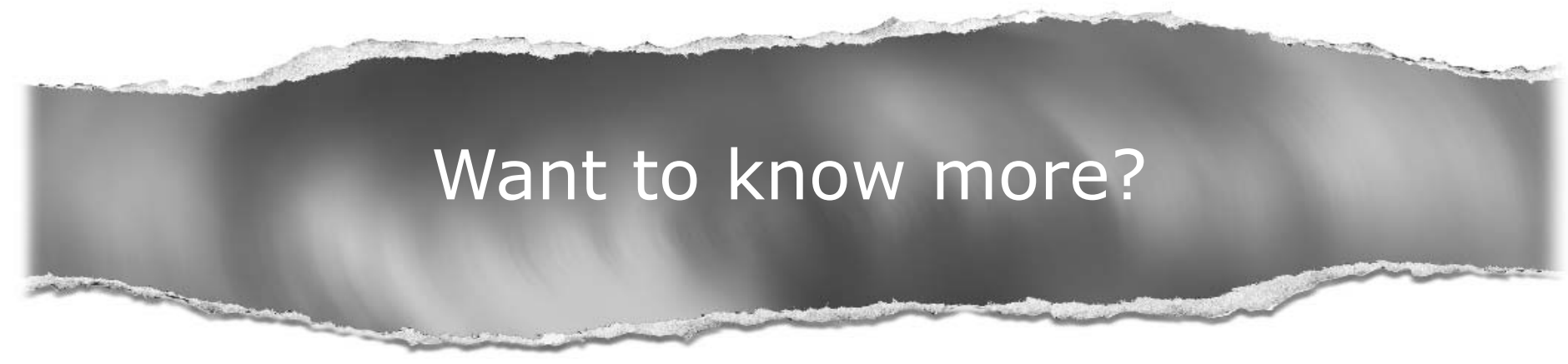

Data supplements

Limited space in printed journals means that interesting data and other material are often edited out of articles; however, limitless cyberspace means that we can include this information online. Look out for additional tables, references, illustrations.

\section{www.archdischild.com}

